\title{
Functorial Aspects of the Space of Generalized Connections*
}

\author{
J. M. Velhinho \\ Departamento de Física, Universidade da Beira Interior \\ R. Marquês D'Ávila e Bolama, 6201-001 Covilhã, Portugal \\ jvelhi@dfisica.ubi.pt
}

\begin{abstract}
We give a description of the category structure of the space of generalized connections, an extension of the space of connections that plays a central role in loop quantum gravity.
\end{abstract}

\section{Introduction}

A central object in loop quantum gravity $[1,2,3]$ is the so-called kinematical Hilbert space. This is the space where basic kinematical operators, as well as quantum versions of the constraints of canonical general relativity, are defined. The kinematical Hilbert space is an $L^{2}$ space of functions on a compact space $\overline{\mathcal{A}}[4,5,6]$, square integrable with respect to the AshtekarLewandowski measure [5]. Properties of the space $\overline{\mathcal{A}}$, known as the space of generalized connections, therefore play an important role in loop quantum gravity.

In this contribution we focus on one particular aspect of the space $\overline{\mathcal{A}}$. As first pointed out by Baez [7], the proper framework to express the algebraic properties of $\overline{\mathcal{A}}$ implicit in earlier formulations $[6,8,9]$ uses the language of category theory. Following previous works $[10,11]$, we present here a concise description of the category structure of $\overline{\mathcal{A}}$. In section 2 we present the space $\overline{\mathcal{A}}$ as a space of functors equipped with a natural topology. In section 3 we

*Presented at the Workshop on Quantum Gravity and Noncommutative Geometry, Universidade Lusófona, Lisbon, July 2004. 
show how extensions of the group of gauge transformations and of the group of diffeomorphisms fit within the category formulation of $\overline{\mathcal{A}}$.

\section{Generalized connections}

\subsection{Groupoid of paths $\mathcal{P}$}

Let $\Sigma$ be an analytic, connected and orientable $d$-dimensional manifold. Let us consider the set $\mathcal{C}$ of all continuous, oriented and piecewise analytic parametrized curves in $\Sigma$, i.e. maps

$$
c:\left[0, t_{1}\right] \cup \ldots \cup\left[t_{n-1}, 1\right] \rightarrow \Sigma
$$

that are continuous in all the domain $[0,1]$, analytic in the closed intervals $\left[t_{k}, t_{k+1}\right]$ and such that the images $c(] t_{k}, t_{k+1}[)$ of the open intervals $] t_{k}, t_{k+1}[$ are submanifolds embedded in $\Sigma$. Maps $s$ and $r$ are defined, respectively, by $s(c)=c(0), r(c)=c(1)$.

Given two curves $c_{1}, c_{2} \in \mathcal{C}$ such that $s\left(c_{2}\right)=r\left(c_{1}\right)$, let $c_{2} c_{1} \in \mathcal{C}$ denote the natural composition given by

$$
\left(c_{2} c_{1}\right)(t)= \begin{cases}c_{1}(2 t), & \text { for } t \in[0,1 / 2] \\ c_{2}(2 t-1), & \text { for } t \in[1 / 2,1]\end{cases}
$$

Consider also the operation $c \mapsto c^{-1}$ given by $c^{-1}(t)=c(1-t)$. The above composition of parametrized curves is not truly associative, since the curves $\left(c_{3} c_{2}\right) c_{1}$ and $c_{3}\left(c_{2} c_{1}\right)$ are related by a reparametrization, i.e. by an orientation preserving piecewise analytic diffeomorphism $[0,1] \rightarrow[0,1]$. Similarly, the curve $c^{-1}$ is not the inverse of the curve $c$. (We refer to compositions of the form $c^{-1} c$ as retracings.)

To achieve a well defined associative composition, with inverse, one considers the following equivalence relation: two curves $c, c^{\prime} \in \mathcal{C}$ are said to be equivalent if $s(c)=s\left(c^{\prime}\right), r(c)=r\left(c^{\prime}\right)$ and $c$ and $c^{\prime}$ coincide up to a finite number of retracings and a reparametrization.

We denote the set of the above defined equivalence classes by $\mathcal{P}$, and refer to generic elements of $\mathcal{P}$ as paths $p$.

The set of paths $\mathcal{P}$ is then naturally equipped with a groupoid structure, as follows. The composition of paths is defined by the composition of elements of $\mathcal{C}$ : if $p, p^{\prime} \in \mathcal{P}$ are such that $r(p)=s\left(p^{\prime}\right)$, one defines $p^{\prime} p$ as the equivalence class of $c^{\prime} c$, where $c$ (resp. $c^{\prime}$ ) belongs to the class $p\left(p^{\prime}\right)$. The composition in $\mathcal{P}$ is now associative, since $\left(c_{3} c_{2}\right) c_{1}$ and $c_{3}\left(c_{2} c_{1}\right)$ belong to 
the same equivalence class. The points of $\Sigma$ play the role of objects in this context. Points are in one to one correspondence with identity paths: given $x \in \Sigma$, the corresponding identity $\mathbf{1}_{x} \in \mathcal{P}$ is the equivalence class of $c^{-1} c$, with $c \in \mathcal{C}$ such that $s(c)=x$. If $p$ is the class of $c$ then $p^{-1}$ is the class of $c^{-1}$. It is clear that $p^{-1} p=\mathbf{1}_{s(p)}$ and $p p^{-1}=\mathbf{1}_{r(p)}$.

One, therefore, has a well defined groupoid, whose set of objects is $\Sigma$ and whose set of arrows is $\mathcal{P}$. As usual, we will use the same notation, $\mathcal{P}$, both for the set of arrows and for the groupoid.

\subsection{Set of functors $\operatorname{Hom}[\mathcal{P}, G]$}

Let $G$ be a (finite dimensional) connected and compact Lie group. Let $\operatorname{Hom}[\mathcal{P}, G]$ denote the set of functors from the groupoid $\mathcal{P}$ to the group $G$, i.e. the set of maps $\bar{A}: \mathcal{P} \rightarrow G$ such that $\bar{A}\left(p^{\prime} p\right)=\bar{A}\left(p^{\prime}\right) \bar{A}(p)$ and $\bar{A}\left(p^{-1}\right)=\bar{A}(p)^{-1}$.

Let us show that the space $\mathcal{A}$ of smooth $G$-connections on any given principal $G$-bundle over $\Sigma$ is a subset of $\operatorname{Hom}[\mathcal{P}, G]$. We assume that a fixed trivialization of the bundle has been chosen. Connections can then be identified with local connection potentials.

The space of connections $\mathcal{A}$ is injectively mapped into Hom $[\mathcal{P}, G]$ through the use of the parallel transport, or holonomy, functions. The holonomy defined by a connection $A \in \mathcal{A}$ and a curve $c \in \mathcal{C}$ is denoted by $h_{c}(A)$. Using the fixed trivialization, one can assume that holonomies $h_{c}(A)$ take values on the group $G$. The following properties of holonomies hold: $i) h_{c}(A)$ is invariant under reparametrizations of $c$; ii $h_{c_{1} c_{2}}(A)=h_{c_{1}}(A) h_{c_{2}}(A)$ and iii) $h_{c^{-1}}(A)=h_{c}(A)^{-1}$. One thus conclude that $h_{c}(A)$ depends only on the equivalence class of $c$, i.e., for fixed $A \in \mathcal{A}, h_{c}(A)$ defines a function on $\mathcal{P}$, with values on $G$. This function is, moreover, a functor, by $i i)$. Summarizing, we have a map $\mathcal{A} \rightarrow \operatorname{Hom}[\mathcal{P}, G]$ :

$$
A \mapsto \bar{A}_{A}, \bar{A}_{A}(p):=h_{p}(A), \forall p \in \mathcal{P} .
$$

That this map is injective is guaranteed by the fact that the set of holonomy functions $\left\{h_{c}, c \in \mathcal{C}\right\}$ separates points in $\mathcal{A}$ [12], i.e. given $A \neq A^{\prime}$ one can find $c \in \mathcal{C}$ such that $h_{c}(A) \neq h_{c}\left(A^{\prime}\right)$.

The set $\operatorname{Hom}[\mathcal{P}, G]$ is, however, much larger than $\mathcal{A}$. To begin with, depending on $\Sigma$ and $G$, different bundles may exist, and Hom $[\mathcal{P}, G]$ contains the space of connections of all these bundles. Moreover, elements of $\operatorname{Hom}[\mathcal{P}, G]$ that do not correspond to any smooth connection do exist $[4,5]$. 


\subsection{Topology}

A natural topology in $\operatorname{Hom}[\mathcal{P}, G]$ can be introduced in several equivalent ways. The one more closely related to well known methods in quantum field theory uses the projective structure of $\operatorname{Hom}[\mathcal{P}, G][5,13,14]$. We follow a different, more direct, approach.

Let us consider the set of all maps from $\mathcal{P}$ to $G$, identified with the product space $\times_{p \in \mathcal{P}} G$. The product space is compact Hausdorff when equipped with the Tychonov topology. We thus let the topology on $\operatorname{Hom}[\mathcal{P}, G] \subset$ $\times_{p \in \mathcal{P}} G$ be the subspace topology. It is clear that Hom $[\mathcal{P}, G]$ then becomes a compact Hausdorff space: the Hausdorff property is inherited from $\times_{p \in \mathcal{P}} G$, and from the fact that $\operatorname{Hom}[\mathcal{P}, G]$ contains only functors follows easily that it is a closed set, therefore compact.

A more explicit characterization of the topology on $\operatorname{Hom}[\mathcal{P}, G]$ is the following: it is the weakest topology such that all maps

$$
\pi_{p}: \operatorname{Hom}[\mathcal{P}, G] \rightarrow G, \quad \bar{A} \mapsto \bar{A}(p),
$$

are continuous, $\forall p \in \mathcal{P}$. The thus obtained compact Hausdorff space is the so-called space of generalized connections, usually denoted by $\overline{\mathcal{A}}$.

\section{Generalized gauge transformations and diffeo- morphisms}

Two groups act naturally and continuously on $\overline{\mathcal{A}}$. One is the group of natural transformations of the set of functors $\operatorname{Hom}[\mathcal{P}, G] \equiv \overline{\mathcal{A}}$. This group is well understood, and is widely accepted as the generalization of the group $\mathcal{G}=C^{\infty}(\Sigma, G)$ of smooth local gauge transformations. The second group of interest is the group $\operatorname{Aut}(\mathcal{P})$ of automorphisms of the groupoid $\mathcal{P}$. It contains the group Diff ${ }^{\omega}(\Sigma)$ of analytic diffeomorphisms as a subgroup. Although extensions of $\operatorname{Diff}^{\omega}(\Sigma)$ are welcome in applications of the current formalism to quantum gravity, the potencial role of the group $\operatorname{Aut}(\mathcal{P})$, or subgroups thereof, is still not clear.

We begin by discussing the group of natural transformations of $\overline{\mathcal{A}}$. In this case, natural transformations form the group, denoted by $\overline{\mathcal{G}}$, of all maps $\mathrm{g}: \Sigma \rightarrow G$, under pointwise multiplication. The action of $\overline{\mathcal{G}}$ on $\overline{\mathcal{A}}$ can be written as $\overline{\mathcal{A}} \times \overline{\mathcal{G}} \ni(\bar{A}, \mathrm{~g}) \mapsto \bar{A}_{\text {g }}$ such that

$$
\bar{A}_{\mathrm{g}}(p)=\mathrm{g}(r(p)) \bar{A}(p) \mathrm{g}(s(p))^{-1} .
$$


This action is readily seen to be continuous. In fact, since the topology on $\overline{\mathcal{A}}$ is the weakest such that all maps $\pi_{p}(2)$ are continuous, one can conclude that a map $\varphi: \overline{\mathcal{A}} \rightarrow \overline{\mathcal{A}}$ is continuous if and only if the maps $\pi_{p} \circ \varphi$ are continuous $\forall p$, which is the case for elements of $\overline{\mathcal{G}}$.

Expression (3) is a generalization of the action of smooth gauge transformations on the set of parallel transport functions $h_{p}(A)$ for smooth connections. It is therefore natural to accept $\overline{\mathcal{G}}$ as the generalized group of gauge transformations on $\overline{\mathcal{A}}$. This extension of the gauge group is in fact required: since $\overline{\mathcal{A}}$ now contains arbitrary functors from $\mathcal{P}$ to $G$, to $\bmod$ out only by smooth gauge transformations would leave spurious degrees of freedom untouched $[6,8,10]$.

Let us now turn to the group $\operatorname{Aut}(\mathcal{P})$ of automorphisms of the groupoid $\mathcal{P}$. By definition, an automorphism of $\mathcal{P}$ is an invertible functor from $\mathcal{P}$ to itself. An element $F \in \operatorname{Aut}(\mathcal{P})$ is therefore characterized by a bijection of $\Sigma$ and a composition preserving bijection on the set of paths, such that $F\left(\mathbf{1}_{x}\right)=\mathbf{1}_{F(x)}, \forall x \in \Sigma$. The action of $\operatorname{Aut}(\mathcal{P})$ on $\overline{\mathcal{A}}$ is given by

$$
\bar{A} \mapsto F \bar{A}: F \bar{A}(p)=\bar{A}\left(F^{-1} p\right), \forall p \in \mathcal{P}, F \in \operatorname{Aut}(\mathcal{P}) .
$$

The continuity of this action is clear, since $\pi_{p} \circ F=\pi_{F^{-1}}$.

The group $\operatorname{Aut}(\mathcal{P})$ contains as a subgroup the natural representation of the group Diff ${ }^{\omega}(\Sigma)$ of analytic diffeomorphisms of $\Sigma$, whose action on curves factors through the equivalence relation that defines $\mathcal{P}$. The group $\operatorname{Aut}(\mathcal{P})$ therefore emerges, in the current context, as the largest possible extension of $\operatorname{Diff}^{\omega}(\Sigma)$. Extensions of $\operatorname{Diff}^{\omega}(\Sigma)$ are welcome in applications to quantum gravity. In fact, the very formalism suggests some sort of extension of Diff ${ }^{\omega}(\Sigma)$. Like in the above mentioned replacement of the classical gauge group $\mathcal{G}$ by $\overline{\mathcal{G}}$, such extension is not fully motivated from the classical perspective, but it is, nevertheless, likely to be required for an appropriate implementation of diffeomorphism invariance, once $\mathcal{A}$ is replaced by $\overline{\mathcal{A}}$.

Several subgroups of $\operatorname{Aut}(\mathcal{P})$ have been proposed so far as candidates to an appropriate extension of Diff ${ }^{\omega}(\Sigma)$. One such extension, including homeomorphisms of $\Sigma$ of class $C^{n}$, appears in a recent report by Ashtekar and Lewandowski [1]. Another recent proposal is due to Fleischhack [15], who considers the so-called stratified analytic diffeomorphisms. Previous approaches allowed for stronger deviation from classical smoothness (on lower dimensional subsets of $\Sigma$ only), like e.g. in Zapata's [16] proposal of replacing Diff ${ }^{\omega}(\Sigma)$ by the group of piecewise analytic diffeomorphisms. (See also $[2,17]$ for related work, in the framework of piecewise smooth curves.) 


\section{Acknowledgements}

I thank the organizers of the Lusófona Workshop on Quantum Gravity and Noncommutative Geometry, Aleksandar Mikovic, Nuno Costa Dias and João Nuno Prata. I am most grateful to José Mourão for discussions. This work was supported in part by POCTI/33943/MAT/2000, POCTI/FNU/49529/2002 and POCTI/FP/FNU/50226/2003.

\section{References}

[1] A. Ashtekar and J. Lewandowski, Background independent quantum gravity: a status report, Class. Quant. Grav. 21 (2004) R53.

[2] C. Rovelli, Quantum Gravity (Cambridge Univ. Press, 2004).

[3] T. Thiemann, Modern Canonical Quantum General Relativity (Cambridge Univ. Press, 2004), at press.

[4] A. Ashtekar and C. J. Isham, Representations of the holonomy algebras of gravity and non-abelian gauge theories, Class. Quant. Grav. 9 (1992) 1433-1468.

[5] A. Ashtekar and J. Lewandowski, Representation theory of analytic holonomy $C^{\star}$ algebras, in Knots and Quantum Gravity, ed. J. Baez (Oxford Univ. Press, 1994).

[6] J. Baez, Generalized measures in gauge theory, Lett. Math. Phys. 31 (1994) 213-224.

[7] J. Baez, Spin networks in gauge theories, Adv. Math. 117 (1996) 253-272.

[8] A. Ashtekar and J. Lewandowski, Differential geometry on the space of connections via graphs and projective limits, J. Geom. Phys. 17 (1995) 191-230.

[9] A. Ashtekar, J. Lewandowski, D. Marolf, J. Mourão and T. Thiemann, Quantization of diffeomorphism invariant theories of connections with local degrees of freedom, J. Math. Phys. 36 (1995) 6456-6493. 
[10] J. M. Velhinho, A groupoid approach to spaces of generalized connections, J. Geom. Phys. 41 (2002) 166-180.

[11] J. M. Velhinho, On the structure of the space of generalized connections, Int. J. Geom. Meth. Mod. Phys. 1 (2004) 311-334.

[12] R. Giles, Reconstruction of gauge potentials from Wilson loops, Phys. Rev. D24 (1981) 2160-2168.

[13] D. Marolf and J. Mourão, On the support of the AshtekarLewandowski measure, Commun. Math. Phys. 170 (1995) 583606.

[14] A. Ashtekar and J. Lewandowski, Projective techniques and functional integration for gauge theories, J. Math. Phys. 36 (1995) 2170-2191.

[15] C. Fleischhack, Representations of the Weyl Algebra in Quantum Geometry, math-ph/0407006.

[16] J. Zapata, Combinatorial space from loop quantum gravity, Gen. Rel. Grav. 30 (1998) 1229-1245.

[17] W. Fairbairn and C. Rovelli, Separable Hilbert space in loop quantum gravity, J. Math. Phys. 45 (2004) 2802-2814. 